\title{
Gastrotórax a tensión como causa de muerte por shock obstructivo. Caso clínico
}

\author{
JUAN F. GARCÍA-REGALADOํ, MARIANA M. NAVARRO-ROJAS ${ }^{1}$ \\ 1. Hospital General de Zona 3, Instituto Mexicano del Seguro Social, México.
}

\begin{abstract}
Tension gastrothorax as a cause of death by obstructive shock - Case report

Introduction: Tension gastrothorax is caused by the herniation of the stomach into the thorax due to a congenital defect of the diaphragm; the Bochdaleck diaphragmatic hernia (HDB) is the most frequent type. Objective: Tension gastrothorax should be considered as a differential diagnosis in patients with obstructive shock and tension pneumothorax. Case report: A previously healthy 10 month-old male infant, who presented increased respiratory distress, increased volume of the left hemithorax, absence of breath sounds, ipsilateral hyper-resonance, $76 \%$ saturation, cold skin and capillary filling $>5$ seconds, followed by a cardio-respiratory arrest. Due to clinical suspicion of pneumothorax, needle decompression was performed reversing cardiac arrest, but with persistent hemodynamic and respiratory instability; chest radiograph suggested diaphragmatic hernia. He underwent surgery confirming the presence of a diaphragmatic hernia of $5 \mathrm{~cm}$. Conclusions: The evolution of this case shows the difficulty differentiating a tension gastrothorax from tension pneumothorax in patients admitted to the emergency room who are in serious condition; therefore, a high index of suspicion is needed for its identification.
\end{abstract}

(Key words: Gastrothorax, diaphragmatic hernia, pneumothorax, shock).

Rev Chil Pediatr 2014; 85 (4): 476-480

\section{RESUMEN}

Introducción: El gastrotórax a tensión se produce por la herniación del estómago hacia el tórax, debido a un defecto congénito del diafragma, siendo el más frecuente la hernia diafragmática de Bochdaleck (HDB). Objetivo: Ejemplificar que, como diagnóstico diferencial del paciente con shock obstructivo y neumotórax a tensión, se debe considerar el gastrotórax a tensión. Caso clínico: Lactante masculino de 10 meses de edad, previamente sano, que evolucionó con dificultad respiratoria aguda, aumento de volumen de hemitórax izquierdo, ausencia de ruidos respiratorios e hiper-resonancia ipsilateral, saturación del $76 \%$, piel fría y

Recibido el 05 de junio de 2013, última versión aceptada para publicación el 25 de junio de 2014.

Potenciales conflictos de interés: Este trabajo cumple con los requisitos sobre consentimiento /asentimiento informado, comité de ética, financiamiento, estudios animales y sobre la ausencia de conflictos de intereses según corresponda.

Correspondencia a:

García-Regalado Juan Francisco.

E-mail: juan.garciare@imss.gob.mx 
llenado capilar mayor de 5 segundos, seguido de un paro cardio-respiratorio. Debido a sospecha clínica de neumotórax se realizó descompresión mediante punción con aguja en conjunto con maniobras, con reversión del paro cardíaco, pero con persistencia de la inestabilidad respiratoria y hemodinámica, radiografía sugerente de hernia diafragmática. Se ingresó a cirugía confirmando la presencia de una hernia diafragmática de $5 \mathrm{~cm}$. Conclusiones: La evolución del presente caso ilustra la dificultad para diferenciar un gastrotórax a tensión de un neumotórax a tensión en el contexto de paciente grave en la sala de urgencias, por lo que debe de tenerse un alto índice de sospecha para su identificación.

(Palabras clave: Gastrotórax, hernia diafragmática, neumotórax, shock).

Rev Chil Pediatr 2014; 85 (4): 476-480

\section{Introducción}

Las hernias diafragmáticas congénitas ocurren en 1 de cada 3.000 nacimientos, el más común de los defectos es el tipo posterolateral (Bochdalek), la incidencia de la herniación es en el $78-90 \%$ posterolateral, 1,5 a $6 \%$ retroesternal vía el foramen de Morgani y 14 a $24 \%$ vía del hiato esofágico. La herniación del lado izquierdo es la más común ya que el hemidiafragma derecho se desarrolla más temprano y el hígado previene la herniación ${ }^{1}$.

Cerca del 90\% de los pacientes se diagnostican en el período prenatal o dentro de las primeras horas de vida con historia de dificultad respiratoria y, sólo del 3 al 30\% se presentarán más allá del período neonatal. A pesar que la mortalidad es baja, la morbilidad es significativa $^{2}$.

El diagnóstico en etapas posteriores resulta un reto debido a la amplia expresión clínica, las cuales resultan en tratamientos inapropiados y riesgo de resultados fatales ${ }^{2}$. Entre los síntomas gastrointestinales se mencionan regurgitaciones, vómitos, pérdida del apetito, constipación, dolor abdominal, diarrea, además de síntomas respiratorios como infecciones pulmonares a repetición, tos, disnea, cianosis y taquipnea ${ }^{3}$. El amplio espectro de síntomas que caracterizan la presentación tardía de una hernia de Bochdaleck explica la dificultad de considerarla como diagnóstico diferencial $^{4,5}$. El objetivo de este reporte fue destacar en el diagnóstico diferencial del paciente con historia de shock obstructivo y clínica de neumotórax a tensión, el gastrotórax a tensión.

\section{Caso clínico}

Lactante varón de 10 meses de edad, producto de un embarazo de 34 semanas de gestación por pre-eclampsia severa, con antecedentes de hipocalcemia durante el primer mes de vida, sin historia de trauma, hospitalizaciones u otras patologías asociadas.

Inició en forma brusca cuadro de $12 \mathrm{~h}$ de llanto incontrolable posterior a alimentación, que evolucionó con cianosis peri-bucal. Presentó paro cardio-respiratorio que revirtió tras 5 minutos a maniobras de reanimación hechas por médico de atención primaria siendo derivado a nuestra unidad.

En Servicio de Urgencia se recibió paciente intubado, sin sedación, con frecuencia respiratoria de $60 / \mathrm{min}$, frecuencia cardíaca $150 / \mathrm{min}$, temperatura $37,5^{\circ} \mathrm{C}$, saturación de oxígeno $76 \%$. Durante la valoración primaria se destacó asimetría torácica secundaria a aumento de volumen del hemitórax izquierdo, ausencia de ruidos respiratorios e hiper-resonancia ipsilateral, piel fría y llene capilar mayor de $5 \mathrm{~s}$. Minutos posteriores a su ingreso presentó nuevo paro cardio-respiratorio que requirió maniobras de reanimación y se diagnosticó neumotórax a tensión. Se realizó descompresión torácica con aguja en segundo espacio intercostal izquierdo con recuperación de la circulación espontánea. Se colocó sonda con drenaje nulo, persistiendo la disminución de murmullo vesicular izquierdo y signos de shock.

Radiografías de tórax sugerentes de hernia diafragmática izquierda con ascenso y distensión aérea a tensión del estómago, compresión $\mathrm{y}$ atelectasia del pulmón ipsilateral; tubo en- 


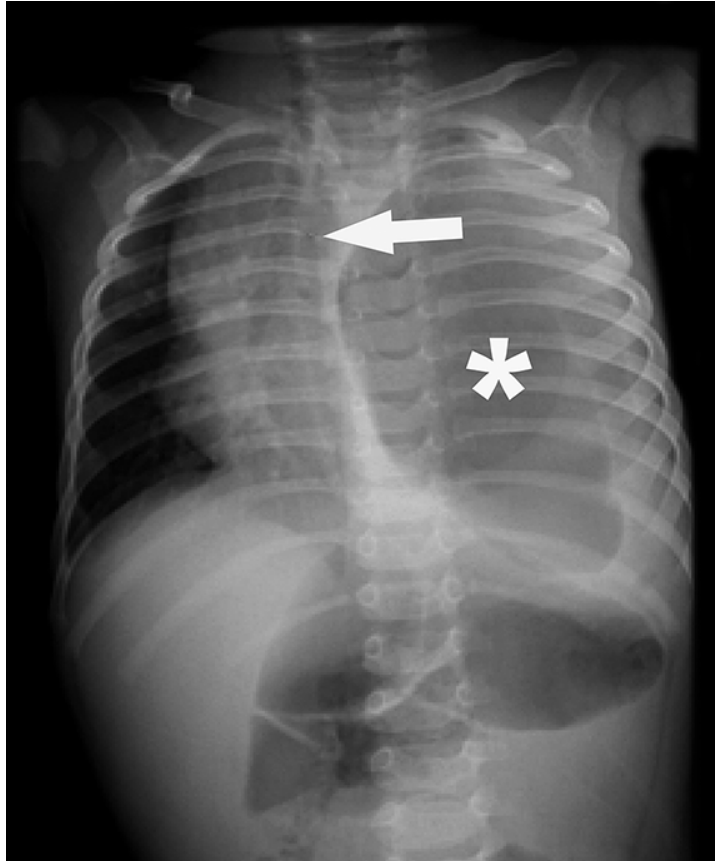

Figura 1. Gran imagen aérea (asterisco) y signos de derrame pleural compatibles con hidroneumotórax a tensión en una radiografía de tórax AP en decúbito supino. Desplazamiento mediastínico importante con desviación de la tráquea a derecha (flecha).

dotraqueal en el bronquio intermedio derecho, con atelectasia secundaria del lóbulo superior e hiperinsuflación de los lóbulos medio e inferior del mismo lado (figuras 1 y 2). Se corrigió colocación de tubo endotraqueal, se inició drenaje por sonda nasogástrica con salida de escaso líquido gástrico, manejo de shock con líquidos y uso aminas tipo dopamina y dobutamina.

Los exámenes de laboratorio de ingreso mostraban leucocitosis, e hipoxemia. Leucocitos $25,9 \mathrm{mil} / \mu \mathrm{l}$, eritrocitos $4,18 \mathrm{milló} / \mu \mathrm{l}, \mathrm{Hb}$ $11,2 \mathrm{~g} / \mathrm{dl}$, Hematocrito 33,8\%, Plaquetas 464 mil/ $\mu \mathrm{l}$; valores gasométricos: $\mathrm{Ph} 7,050 ; \mathrm{PcO}_{2}$ : $34,4 \mathrm{~mm}$ de $\mathrm{Hg} ; \mathrm{PO}_{2} 74,3 \mathrm{~mm}$ de $\mathrm{Hg} \mathrm{HCO}_{3}$ 9,7 mmol/1; $\mathrm{CO}_{2}$ 10,8; Exceso de base -20,7; Saturación de $\mathrm{O}_{2}: 87,2 \%$.

Debido a estado de shock persistente y dificultades para la ventilación mecánica, se decidió pasar a pabellón de urgencia. Durante la cirugía presentó nuevo paro cardio-respiratorio con retorno a la circulación espontánea. En

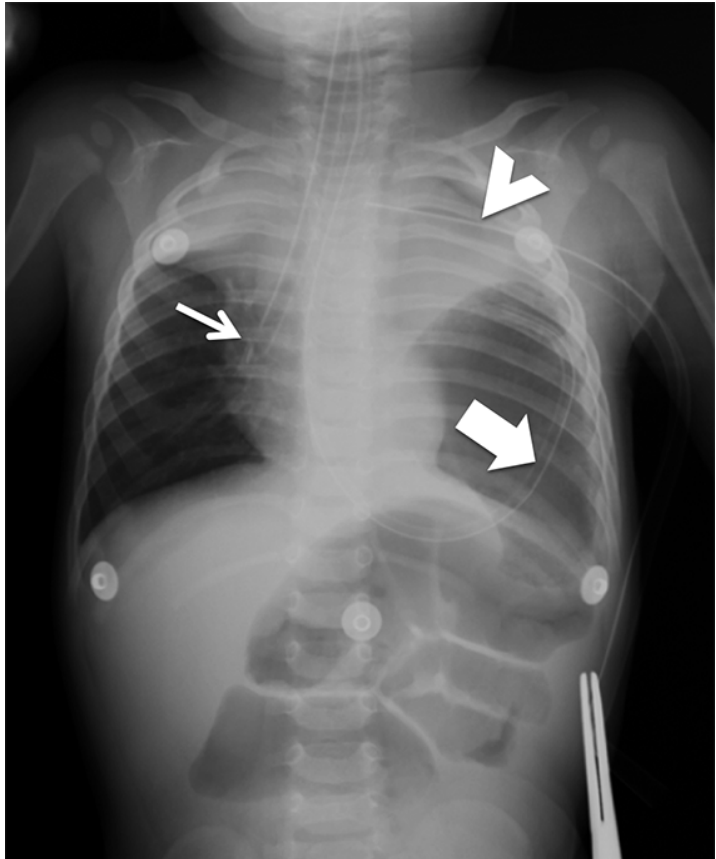

Figura 2. Monointubación con tubo traqueal en bronquio intermedio (flecha fina). Sonda nasogástrica con trayecto transesofágico hasta el abdomen desde donde se dirige hacia el hemitórax izquierdo (flecha gruesa). Drenaje pleural apical izquierdo (cabeza de flecha). Extremo distal de SNG dentro de imagen aérea en base pulmonar izquierda que corresponde a estómago intratorácico. Desplazamiento superior y atelectasia del parénquima pulmonar izquierdo. Atelectasia de lóbulo superior derecho secundario a monointubación. Comparativamente con figura 1 , se observa una mejor alineación mediastínica.

la cirugía se encontró hernia diafragmática izquierda de $5 \mathrm{~cm}$, con eventración torácica de bazo y colón, se realizó cierre primario con parche de Graham.

El paciente fue trasladado a Hospital de tercer nivel para manejo de terapia intensiva donde falleció después de $5 \mathrm{~h}$.

\section{Discusión}

Nuestro caso se ajusta a la epidemiología descrita para las hernias diafragmáticas congénitas $^{6}$ siendo la herniación postero-lateral izquierda la más frecuente, con presentación 2:1 en hombres. Las manifestaciones clínicas varían dependiendo del tipo de órganos abdominales que se hernian hacia el tórax. 
El gastrotórax a tensión es una rara complicación de las hernias diafragmáticas que ocurre cuando el estómago se hernia a través de un defecto en el diafragma hacia el tórax, se distiende con aire y lleva a compromiso hemodinámico. Es visto más frecuentemente en el período perinatal o posterior a trauma abdominal. Debido a las similitudes clínicas y radiológicas entre neumotórax y gastrotórax a tensión se debe de tener un alto índice de sospecha ya que usualmente es confundido con un neumotórax a tensión 7 .

Los hallazgos físicos son similares al neumotórax a tensión e incluyen taquicardia, hipotensión, hipoxia, distensión yugular, disminución o ausencia de los ruidos respiratorios, hiper-resonancia del tórax ipsilateral. La radiografía puede mostrar una estructura con nivel hidro-aéreo causando constricción apical del pulmón.

La inserción de un tubo pleural puede aliviar los síntomas en forma temporal, pero resultar en consecuencias serias que dañen las vísceras abdominales ${ }^{2,8}$. Se ha descrito que entre las complicaciones de confundir una hernia con un neumotórax se encuentran la lesión a los órganos abdominales a nivel gástrico e intestinal, lo que se asocia a una elevada morbimortalidad $^{3}$.

Este caso se presentó en el contexto del servicio de urgencias de un hospital de segundo nivel, en un lactante con cuadro de inestabilidad hemodinámica y respiratoria, clínicamente compatible con neumotórax a tensión. Siguiendo esta hipótesis, el paciente fue manejado de acuerdo a los protocolos del American Trauma Life Support (ATLS) que mencionan que frente a la sospecha de un paciente con neumotórax a tensión se realice la descompresión torácica sin necesidad de tener radiografía previa $^{9}$, y a los algoritmos del Pediatric Advanced Life Support (PALS) ${ }^{10}$, que consideran el neumotórax como dentro del apartado de las " $T$ 's" del diagnóstico diferencial una de las causas reversibles de paro cardio-respiratorio (Toxinas, Taponamiento cardíaco, Trombosis, Traumatismo, neumotórax a Tensión), que requiere resolución inmediata para lograr el retorno a la circulación espontánea.

A pesar que la literatura menciona que el gastrotórax debe de ser uno de los diagnósticos diferenciales a considerar en todo lactante con cuadro clínico sugerente, como ocurrió en el caso presentado, consideramos que el manejo en la sala de urgencias ante un paciente en paro cardio-respiratorio con clínica sugestiva de un neumotórax a tensión es la descompresión torácica apegado a los algoritmos de manejo del paciente en consideración al alto riesgo de muerte ante una causa potencialmente reversible mediante medidas relativamente sencillas.

El diagnóstico diferencial con el gastrotórax a tensión con inestabilidad hemodinámica severa en la sala de urgencias es extremadamente difícil, especialmente en el caso descrito, en que no se contaba con antecedentes, exámenes previos, y los protocolos de manejo no consideran esta posibilidad.

El diagnóstico del gastrotórax se realiza usualmente por medio de una radiografía de tórax y se confirma con una tomografía de tórax ${ }^{11}$ situaciones que en el contexto de un paciente bajo reanimación no pueden ser realizadas. El presente caso demuestra que en todo lactante con presentación similar se debe de atento ante esta posibilidad, y la búsqueda del gastrotórax como parte del protocolo de abordaje diferencial, en especial, en los lactantes en los que sea posible realizar los exámenes de urgencia necesarios.

\section{Conclusión}

Nuestro paciente se presentó con gastrotórax a tensión y tal como lo han descrito algunos reportes de casos, el manejo como neumotórax ${ }^{1,4}$ lo llevó a tener mejoría transitoria de la sintomatología, manifiesto por el retorno a la circulación espontánea. Por lo anteriormente expuesto, consideramos que todo lactante que se presente en la sala de urgencias con clínica de neumotórax debe de considerarse la posibilidad de gastrotórax y realizarse radiografía de tórax como parte del abordaje inicial.

\section{Referencias}

1.- Juwarkar CS, Kamble DS, Sawant V: A late presenting congenital diaphragmatic hernia misdiagnosed as spon- 
taneous pneumothorax. Indian Journal of Anesthesia 2010; 54 (5): 464-6.

2.- Sridhar $A V$, Nichani $S$ : Late presenting congenital diaphragmatic hernia. Emerg Med J 2004; 21: 261-2.

3.- Zaleska-Dorobisz U, Bagłaj M, Sokołowska D, Ładogórska J, Moroń K: Late presenting diaphragmatic hernia: Clinical and diagnostic aspects. Med Sci Monit 2007; 13: 137-43.

4.- Moilia F, Salvatore A, Gendalina G, Palazzo E, Rancati $A$, Zoja R: Lethal Bochdalek hernia in a three-year-old: pathological findings and medicolegal investigation in accusation of malpractice. Med Sci Law 2013; 53: 51-4.

5.- Baeza C, Velázquez H, Vidrio F: Complicaciones tardías imprevistas de las hernias diafragmáticas. Acta Pediátrica de México 2011; 24: 319-22.

6.- Baglaj M: Late-presenting congenital diaphragmatic hernia in children: a clinical spectrum. pediatr Surg Int 2004; 20 (9): 658-69.

7.- Hooker R, Claudius I, Truong A: Tension Gastrothorax in a child presenting with abdominal pain. West $\mathrm{J}$ Emerg Med 2012; 13 (1): 117-8.

8.- Patton JM, González J, Dillard TA, Szerlip HM: Hyperlucent left Hemithorax and respiratory distress. Chest 2008; 134: 872-5.

9.- American College of Surgerons Commitee of Trauma: Thoracic Trauma. En: Advanced Trauma Life Support 2008; 85-100.

10.- American Heart Association: Reconocimiento y manejo del paro cardíaco. En: Soporter Vital Avanzado Pediátrico: Editorial Integracolor Ltd, 2012; 141-70.

11.- Berríos C, Melipillán A: Caso clínico-radiológico pediátrico. Rev Chil Enf Respir 2010; 26: 158-60. 